\section{Resistencia de las suturas no absorbibles, con punto simple, post-extracción de cordales}

\author{
Resistance of non-absorbable sutures, with simple point, post- \\ extraction of cords
}
Resistência de suturas não absorvíveis, com ponto único, pós- extração de cordões

\author{
Gabriel Díaz \\ gdiaz021@gmail.com \\ Saribel Seijas \\ sariseijas1992@gmail.com \\ Recibido enero 2018 / Revisión febrero 2018 / Aceptado 1 de mayo 2018
}

\section{RESUMEN}

Introducción: En la evolución del material de sutura, se incluyen desde los diseños para procedimientos quirúrgicos específicos hasta las que facilitan el cierre de las heridas para disminuir la infección postoperatoria. Objetivo: Determinar la resistencia de suturas no reabsorbible, con punto simple, post-extracción de cordales, en incisiones lineales, en los pacientes atendidos en el Centro de Especialidades Médicas-Odontológicas en Venezuela. Materiales y métodos: Se realizó una investigación de campo, de nivel descriptivo, con una revisión literaria sustentada en la operacionalización de variables para el mejor análisis de los datos. Resultados: La resistencia de la sutura no absorbible, tipo Nylon fue efectiva en un $90 \%$. Se evidenció la pérdida leve de la fuerza de tensión de la sutura también en un $90 \%$ y un cierre alveolar en un $100 \%$ en promedio de 8 días. Conclusión: La resistencia de la sutura no reabsorbible tipo Nylon, evita el ingreso de alimentos en la zona tratada, manteniendo una buena higiene y facilitando el adecuado proceso de cicatrización, minimizando el trauma excesivo e impidiendo la inflamación y el edema, siempre apoyándose en la correcta aplicación de la técnica de sutura para el éxito y la evolución excelente del procedimiento.

Palabras claves: suturas; nylon; procedimientos quirúrgicos orales; cirugía bucal

\section{ABSTRACT}

Introduction: In the evolution of the suture material, designs are included for specific surgical procedures to those that facilitate the closure of wounds to reduce postoperative infection. Objective: To determine the resistance of sutures not reabsorbable with simple point, post-extraction of wisdom teeth, in linear incisions, in the patients attended in the Center of Medical-Odontological Specialties in Venezuela. Materials and methods: A Field, descriptive, research was carried out, with a literary review based on the operationalization of variables for the best analysis of the data. Results: The resistance of the nonabsorbable suture Nylon type was effective in $90 \%$. The slight loss of the tensile strength of the suture was also shown in $90 \%$ and an alveolar closure in $100 \%$ in average of 8 days. Conclusion: The resistance of the nonabsorbable suture Nylon type, prevents the entry of food into the treated area, maintaining good hygiene and facilitating the proper healing process, minimizing excessive trauma and preventing inflammation and edema always relying on the correct application of the suture technique for the success and the excellent evolution of the procedure.
GD: Universidad José Antonio Páez. Facultad de Ciencias de la Salud. Escuela de Odontología. Venezuela.

SS: Universidad José Antonio Páez. Facultad de Ciencias de la Salud. Escuela de Odontología. Venezuela.

Key words: sutures; nylons; oral surgical procedures; surgery, oral 


\section{RESUMO}

GD: Universidad José Antonio Páez. Facultad de Ciencias de la Salud. Escuela de Odontología. Venezuela.

SS: Universidad José Antonio Páez. Facultad de Ciencias de la Salud. Escuela de Odontología. Venezuela.
Introdução: Na evolução do material de sutura, eles são incluídos desde os desenhos de procedimentos cirúrgicos específicos até aqueles que facilitam o fechamento de feridas para reduzir a infecção pós-operatória. Objetivo: Determinar a resistência de suturas não absorvíveis, com um único ponto, pós-extração de cordas, em incisões lineares, em pacientes atendidos no Centro de Especialidades Médico-Dentárias da Venezuela. Materiais e métodos: Foi realizada uma investigação descritiva em campo, com revisão literária baseada na operacionalização de variáveis para a melhor análise dos dados. Resultados: A resistência da sutura não absorvível tipo Nylon foi efetiva em $90 \%$. A leve perda de força de tensão da sutura também foi observada em $90 \%$ e um fechamento alveolar em 100\%, em média, em 8 dias. Conclusão: A resistência do fio de nylon não absorvível, impede a entrada de alimentos na área tratada, mantendo boa higiene e facilitando o processo de cicatrização, minimizando o trauma excessivo e prevenindo inflamações e edemas, sempre contando com a aplicação correta da técnica de sutura para obter sucesso e excelente evolução do procedimento.

Palavras-chave: suturas; nylons; procedimentos cirúrgicos bucais; cirurgia bucal

\section{INTRODUCCIÓN}

$\mathrm{E}$ l uso de la seda negra, para la sinéresis de heridas quirúrgicas, en cavidad bucal es muy común; pero una de sus desventajas es el acumulo de placa bacteriana, principal causante de enfermedades periodontales (1). El éxito de un tratamiento bucal, se debe en gran parte a un control minucioso de esa placa bacteriana. Por lo tanto, los materiales utilizados deben acumular la menor cantidad de placa, siendo el hilo de sutura uno de los materiales más importantes $(2,3)$. De allí, que en la extracción dental como procedimiento quirúrgico para sacar un elemento dental propio del alveolo, se tomen en cuenta todos los demás medios de curación del diente, siendo esta última elección terapéutica, a menos que las condiciones ameriten que sea la opción principal (4).

Además, otros autores argumentan que existen indicaciones para la exodoncia a nivel de los dientes temporales y permanentes como son las caries y la enfermedad periodontal. De hecho, deben considerarse otras causas, como: dientes retenidos, supernumerarios, anomalías de posición y situación, dientes temporales, fracturas, lesiones avanzadas, dientes relacionados con quistes y tumores (5).

De tal manera, que hay que considerar en la extracción dentaria, la sutura o hilo utilizado para ligar los vasos sanguíneos o afrontar los tejidos. Estas se han descritas desde hace mucho - 2.000 a.C.- señalando el uso de cuerdas y tendones de animales como suturas. A través del tiempo, se ha utilizado una amplia variedad de materiales: seda, lino, algodón, pelo de caballo, tendones e intestinos de animales, y alambre de metales preciosos en los procedimientos quirúrgicos (6). También, en el papiro de Edwin Smith en el siglo XVI a.C., se registra quizá la primera descripción del empleo de una sutura al referirse al uso de cuerdas y tendones de animales para ligar y suturar. Además, Joseph Lister introdujo el catgut que se originaba del intestino de gato. Actualmente se realiza de ovinos y bovinos y William Halsted utilizó por primera vez la seda en las intervenciones quirúrgicas.

Por lo que se planteó como propósito de la investigación, determinar la resistencia de las suturas no reabsorbible, con punto simple, post-extracción dentaria en incisiones lineales, en los pacientes 
atendidos en el Centro de Especialidades Médicas Julia Benítez, en el área Odontología en el Municipio Guacara Estado Carabobo.

\section{Clasificación de las suturas}

Las suturas se pueden clasificar de diversas formas: Absorbibles y no absorbibles (naturales y sintéticas). Por su origen (animal, vegetal, mineral, sintéticos). Por su estructura (monofilamento y multifilamento).

Los monofilamentos tienen por característica la facilidad de pasar en los tejidos, resistente a microorganismos y fáciles de anudar.

Las suturas multifilamentos tienen mayor fuerza de tensión y flexibilidad, pueden estar recubiertas y facilitar el paso a través del tejido.

Las suturas absorbibles pueden utilizarse para mantener los bordes de la herida aproximados temporalmente, hasta que haya cicatrizado lo suficiente para soportar la tensión normal. Se preparan con colágeno de mamíferos sanos o con polímeros sintéticos. Algunas se absorben rápidamente $\mathrm{y}$ otras son tratadas, $\mathrm{o}$ químicamente estructuradas para prolongar el tiempo de absorción. Pueden también estar impregnadas o recubiertas con agentes que mejoran sus propiedades de manejo y teñidas con un colorante aprobado por la FDA, para aumentar la visibilidad en el tejido (4).

Las suturas absorbibles naturales son digeridas por enzimas del organismo que atacan y degradan el hilo de sutura. Las suturas sintéticas absorbibles son hidrolizadas, este es un proceso donde penetra gradualmente agua en los filamentos de la sutura ocasionando degradación de la cadena del polímero. En comparación con la acción enzimática de las suturas absorbibles naturales, la hidrólisis tiene como resultado un menor grado de reacción.
Las suturas no absorbibles son aquellas que no son digeridas por las enzimas del organismo o hidrolizadas en el tejido, están compuestas de filamento único o múltiple de metal, sintéticos, o fibras orgánicas que se reducen a un hilo torcido, o trenzado.

Las suturas absorbibles se clasifican en Naturales: Catgut simple, Catgut crómico. Ambos consisten en hilos procesados de colágena altamente purificada, consisten en tiras procesadas de la capa submucosa del intestino de oveja o la capa serosa del intestino de bovino, son hiladas y pulidas electrónicamente en hebras de monofilamento. El simple, en tejidos que cicatrizan rápidamente como membranas mucosas y el tejido subcutáneo, 70 días. El crómico se utiliza en zonas que cicatrizan más lentamente, ejemplo: nivel genitourinario, 90 días (4). Sintéticos: Ácido poliglicólico o poliglactina 910, extremadamente inertes y poseen una gran fuerza de estiramiento, puede ser utilizada en casi todos los tejidos. Absorción rápida en 42 días y el de absorción media en 56 - 72 días.

Polidiaxona: Formada por el poliéster poli (p-dioxanona) blando, flexible de baja afinidad por microrganismos y la absorción es esencialmente completa entre 91 y 119 días.

Poliglecaprone 25: Monofilamento, mayor flexibilidad para fácil manejo $\mathrm{y}$ anudado. Se utiliza en casos donde se requiere gran fuerza tensional al inicio y que disminuya a las dos semanas siguientes de la intervención; tiene una reabsorción entre los 91 y 119 días.

\section{Clasificación de las suturas no absorbibles}

Se clasifican en naturales y sintéticos. Naturales: Seda: ampliamente utilizada y reúne las cualidades de fortaleza, flexibilidad y fácil manejo, puede 
emplearse en una amplia variedad de tejidos que van del oftálmico al cardiovascular. Sintéticos tales como: Nylon: Primordialmente empleada para el cierre de la piel, procedimientos oftálmicos y microcirugía. Disponible en hebras monofilamentosas o entrelazadas, de poliamida 6 y 66, posee gran fuerza de estiramiento y resiste la acción capilar. Polipropileno: Es la más fuerte de todas las suturas, a excepción del acero inoxidable, normalmente se presenta en forma monofilamentosa, de Estereoisómero cristalino isotáctico de polipropileno, pudiendo estar recubierta de teflón. Fibra de poliéster: Tiras de poliester no tratadas, multifilamento, hay sin recubrimiento (Mersilene) y con recubrimiento de polibutilato (Ethibond), es utilizado para prótesis sintéticas vasculares. Acero inoxidable: Ausencia de elementos tóxicos, flexibilidad y calibre fino. Tensión elevada con el mono y multifilamento. Para cierre abdominal, esternón, ortopedia, neurocirugía, reducción de fracturas óseas.

Con este estudio, se beneficiarán investigadores y personas relacionadas con la Odontología, para aplicar los conocimientos en la consulta, en el acto quirúrgico y así beneficiar al paciente, tomando en cuenta resultados a corto plazo.

\section{MATERIALES Y MÉTODOS}

$\mathrm{S}$ e realizó una investigación de campo, con un nivel descriptivo, se miden de forma independiente las variables.
La población estuvo representada por 30 pacientes, de los cuales fue seleccionada una muestra de 10 pacientes que requerían de una cirugía bucal que ameritaba de cicatrización por segunda intención, los cuales fueron atendidos con previo consentimiento informado en el área de Odontología en el Centro de Especialidades Médicas-Odontológicas Julia Benítez, de Guacara, Edo. Carabobo. La muestra fue de tipo probabilístico intencional.

Las técnicas de recolección de datos fueron la historia clínica y una guía de observación en donde se visualizó la evolución del paciente, antes y después que se realizará la sutura. Al mismo tiempo se efectuó, el consentimiento informado, procedimiento que garantiza que el sujeto ha expresado voluntariamente su intención de participar en la investigación (7). Los pacientes se estudiaron durante el acto quirúrgico y durante los controles postquirúrgicos.

\section{RESULTADOS}

$\mathrm{E}$ n función del proceso de estudio pre y post quirúrgico llevado a cada uno de los 10 pacientes y en correspondencia con la recolección de datos, donde se visualizó la evolución del paciente, antes y después que se realizara la sutura, se alcanzaron los siguientes resultados: 


\section{Resistencia de la sutura Post - Quirúrgico}

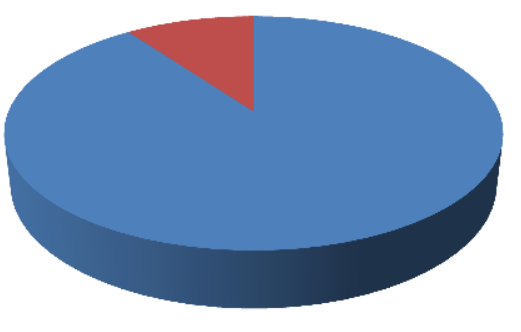

- 90\% - Post Quirurgico.

$10 \%$ - Post Quirurgico.

Gráfico 1. Resistencia de las suturas no absorbibles, con punto simple, en extracción de cordales.

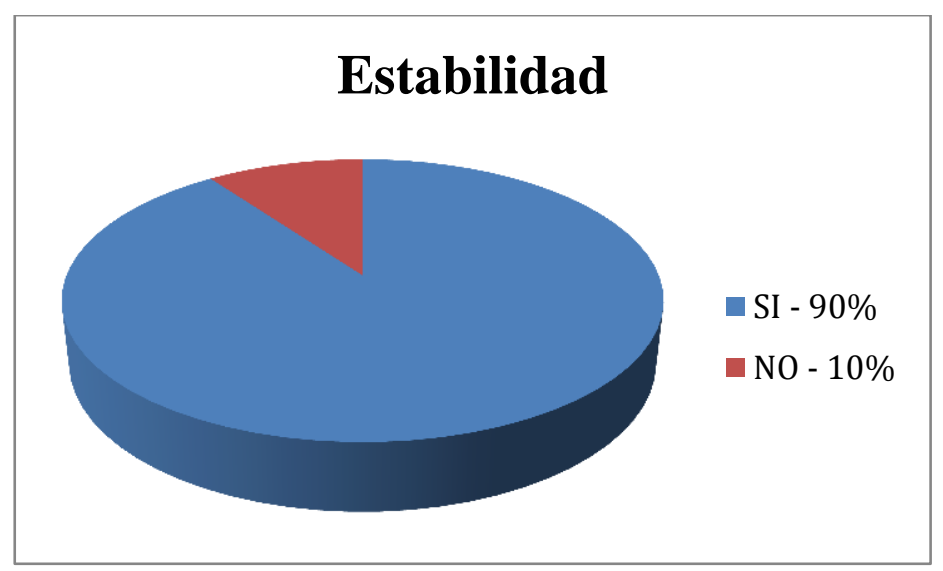

Gráfico 2. Estabilidad de las suturas no absorbibles, con punto simple, en extracción de cordales, en incisiones lineales.

La estabilidad de la sutura se comprobó ser mayor con sutura no reabsorbible con un resultado positivo de un 90\%, es decir en 9 pacientes conservó la estabilidad, mientras que uno sólo no obtuvo los resultados esperados.

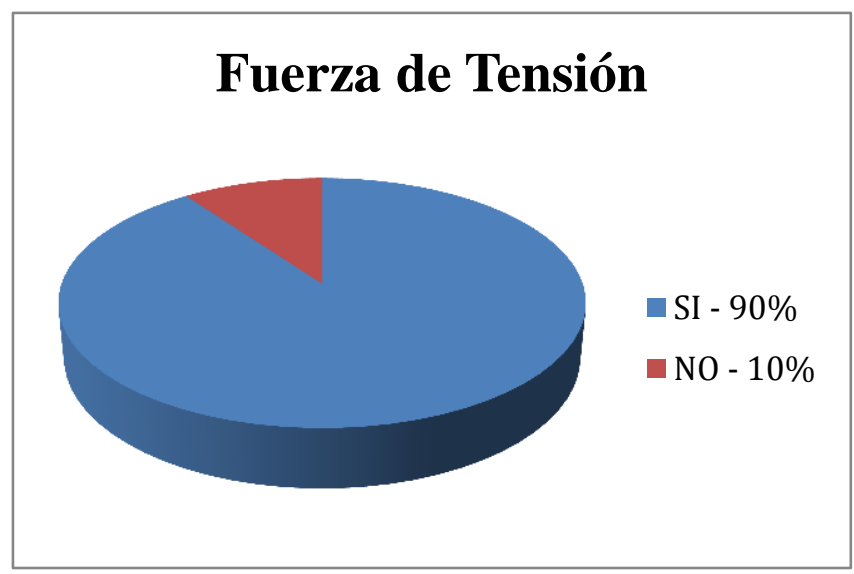

Gráfico 3. Fuerza de tensión de las suturas no absorbibles, con punto simple, en extracción de cordales, en incisiones lineales. 


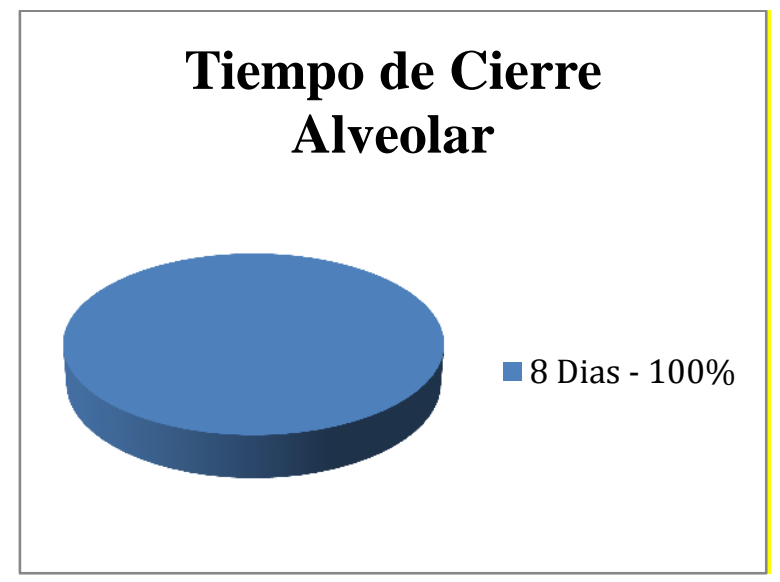

Gráfico 4. Cierre alveolar con suturas no absorbibles, con punto simple, en extracción de cordales, en incisiones lineales.

En cuanto al tiempo de cierre alveolar, este resultó ser en un $100 \%$ de la población de 8 días, representando un menor riesgo post quirúrgico. La fuerza de tensión de la sutura resultó ser mayor con sutura no reabsorbible con un resultado positivo de un $90 \%$ es decir, en 9 pacientes conservó la estabilidad mientras que uno sólo, no obtuvo los resultados esperados.

\section{DISCUSIÓN}

$\mathrm{L}$ as causas por las que se indica una extracción dentaria pueden variar: por prevención, antes de un tratamiento de ortodoncia para evitar apiñamientos, en caso de dolor local o irradiado, presencia de caries, pericoronaritis, problemas periodontales, rizólisis de piezas vecinas, formación de quistes y tumores, alteraciones en la ATM, fracturas dentales debido a inadecuadas maniobras por parte del odontólogo, al momento de la exodoncia. También, está indicada en pacientes que van a ser sometidos a terapias de quimioterapia o radioterapia $\mathrm{y}$ en pacientes que son remitidos por algún tipo de medicina alternativa. Además, los terceros molares con mayor frecuencia se encuentran incluidos, constituyendo un apartado importante de la patología odontológica, no sólo por su frecuencia y su variedad de presentación, sino también por la patología y accidentes que desencadenan, lo que explica que su extracción sea la intervención que realizan más comúnmente los cirujanos orales y maxilofaciales (8-11). Se usó sutura no absorbible de origen sintético (Nylon), aplicado a todos los pacientes que fueron sometidos a procedimientos quirúrgicos, que ameritaron incisiones lineales. Información que sirvió para la determinación de los cambios clínicos de los tejidos, en el área tratada.

Asimismo, se realizaron evaluaciones post-quirúrgicas para visualizar alteraciones de los tejidos y zonas adyacentes y así garantizar el éxito del proceso de cicatrización llevándose a cabo en forma metodológica la técnica de sutura aplicada correctamente sobre los tejidos dentales, que permitió definir y determinar un correcto y acertado uso del material de sutura de acuerdo a una determinada incisión lineal:

Resistencia: Capacidad de soportar presiones $y$ fuerzas aplicadas sin quebrarse, deformarse o sufrir deterioros. Estabilidad: Mantener el equilibrio no cambia o permanece en el mismo lugar 
durante mucho tiempo igual $\mathrm{y}$ una modificación razonablemente pequeña de las condiciones iníciales que no altera significativamente el futuro de la situación. Fuerza de Tensión: Acción que puede modificar el estado de reposo o de movimiento de un cuerpo es decir, puede acelerar o modificar la velocidad, la dirección o el sentido del movimiento de un cuerpo dado. La tensión, por su parte, es el estado de un cuerpo sometido a la acción de fuerzas opuestas que lo atraen. Cierre del Alveolo: Proceso que se da mediante la cicatrización con un fin regenerador, donde a través del epitelio y complejos celulares de la zona se forma un nuevo tejido, con el fin de restaurar la integridad física del mismo.

Estas cualidades clínicas fueron evaluadas en las técnicas de sutura empleadas $y$ se observaron ciertas variaciones y complicaciones dentro de la población en estudio. Descripciones plasmadas en los gráficos, que ilustran los resultados. La resistencia de la sutura no absorbible, tipo Nylon, fue efectiva en un $90 \%$ y se tradujo en el cierre correcto de los tejidos tras la técnica aplicada, evitando en gran medida la pérdida de la fuerza de tensión, luego de los días posteriores al acto quirúrgico. Existió una retención mecánica durante la aplicación de la técnica en un $10 \%$ de los casos al pasar el material entre los tejidos, dificultando la técnica de sutura, así como también se aplicó una fuerza de tensión considerable al observar que la estabilidad de la sutura tendía a variar en el 70\% de los casos tras la fuerza aplicada, siendo una característica de este material al momento de emplear la técnica de sutura, es decir, que se requiere más fuerza para obtener una tensión y estabilidad adecuada.

En conclusión, la resistencia de la sutura no absorbible, tipo Nylon, fue efectiva en un $90 \%$ y se tradujo en el cierre correcto de los tejidos posterior a la aplicación de la técnica aplicada, evitando en gran medida la pérdida de la fuerza de tensión, luego de los días posteriores al acto quirúrgico, la estabilidad de la sutura tiende a variar en el $70 \%$ de los casos, tras la fuerza aplicada, siendo una característica de este material al momento de emplear la técnica de sutura, es decir, que se requiere más fuerza para obtener una tensión y estabilidad adecuada.

En el control postoperatorio, se evidenció la pérdida leve de la fuerza de tensión de la sutura en un $90 \%$, y en un $10 \%$ pérdida completa del punto de sutura. Aunado a un cierre alveolar en un $100 \%$, en promedio de 8 días, donde los tejidos estuvieron aptos para retirar los puntos. En relación a los cambios clínicos de los tejidos, se observó disminución del edema y enrojecimiento de la zona.

Finalmente, el nivel de resistencia del material de sutura no reabsorbible, tipo Nylon, en incisiones lineales, con punto simple, es un material que evita el ingreso de los alimentos a la zona afectada, manteniendo una buena higiene $\mathrm{y}$ facilitando el adecuado proceso de cicatrización, para minimizar el trauma excesivo y evitando la inflamación y el edema, siempre apoyados en la correcta aplicación de la técnica de sutura para el éxito y evolución excelente del procedimiento.

\section{REFERENCIAS}

1. Montilla O.Tratado de cirugía oral y maxilofacial. Tomo I. Madrid: Ediciones S. Larán; 2004

2. Escoda C. Tratado de cirugía bucal. Tomo I. [s.I.]: Editorial Ergón; 2005

3. Freudenberg $\mathrm{S}$. The improvised atraumatic suture: a costreducing technique, not Orly for the topics? Trop Doct. 2001; 31:166-7

4. Fuller, J. Instrumentación quirúrgica. Principios y práctica. [s.I.]: Segunda Editorial; 1988

5. Guevara, J. Principios de cirugía. [s.l.]: Guía básica: Edamex; 2011

6. Puerto $M$, Casas Insua $L$, Cañete $R$. Terceros molares retenidos, su comportamiento en Cuba. Revisión de la literatura. Rev. med. electron. [Internet]. 
2014 [citado 201709 diciembre]; 36 (Suppl 1): 752-762 Disponible en: http://scielo.sld.cu/scielo.php?script=sci_artte xt\&pid=S1684-

1824201418242014000700008\&lng=es

7. Chaparro A, Pérez S, Valmaseda C. Morbilidad de la extracción de los terceros molares en pacientes entre los 12 y 18 años de edad. Med. Oral Patol. Oral Cir Bucal (Ed. impr.) [Internet]. 2005 [citado 201709 diciembre]; 10 (5): 422-431. Disponible en: http://scielo.isciii.es/scielo.php?script=sci_artt ext\&pid=S169844472005000500007\&lng=es

8. Felzani R. "Sutura de los tejidos en el área de cirugía bucal. Revisión de la literatura.
Acta odontol. venez [Internet]. 2007 [citado 201709 diciembre]; 45(4): 598-609. Disponible en: http://ve.scielo.org/scielo.php?script=sci_ arttext\&pid=S000163652007000400018\&lng=es

9. Felzani R. Cicatrización de los tejidos con interés en cirugía bucal. Acta odontol. venez. 2005; 43(3)

10. Donado M. Cirugía bucal. Patología y técnica. 2 ed. Barcelona: III Masson; 2001

11. Rodríguez O, Pérez M. Síntesis de heridas bucofaciales con aplicación de tisuacryl. Rev Cubana Estomatol. 2003; 42(3). Disponible en: http://bvs.sld.cu/revistas/est/vol

- Los autores expresan no poseer conflictos de interés.

- Este estudio fue realizado a través de financiamiento propio.

- Apegado a las más estrictas normas bioéticas según tratado de Helsinki 